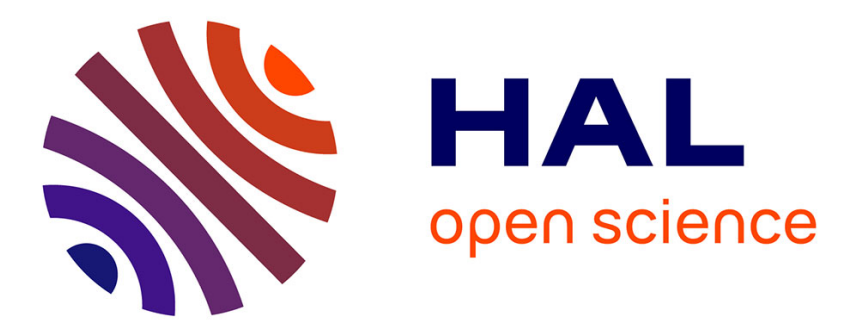

\title{
A Parametric TDoA Technique in the IoT Localization Context
}

\author{
Ahmed Abdel Ghany, Bernard Uguen, Dominique Lemur
}

\section{To cite this version:}

Ahmed Abdel Ghany, Bernard Uguen, Dominique Lemur. A Parametric TDoA Technique in the IoT Localization Context. WPNC, Oct 2019, Bremen, Germany. hal-02302985

\section{HAL Id: hal-02302985 \\ https://hal.science/hal-02302985}

Submitted on 1 Oct 2019

HAL is a multi-disciplinary open access archive for the deposit and dissemination of scientific research documents, whether they are published or not. The documents may come from teaching and research institutions in France or abroad, or from public or private research centers.
L'archive ouverte pluridisciplinaire HAL, est destinée au dépôt et à la diffusion de documents scientifiques de niveau recherche, publiés ou non, émanant des établissements d'enseignement et de recherche français ou étrangers, des laboratoires publics ou privés. 


\section{A Parametric TDoA Technique in the IoT Localization Context}

\author{
Ahmed Abdel Ghany \\ IETR \\ University of Rennes 1 \\ Rennes, France \\ ahmed.abdelghany@univ-rennes1.fr
}

\author{
Bernard Uguen \\ IETR \\ University of Rennes 1 \\ Rennes, France \\ bernard.uguen@univ-rennes1.fr
}

\author{
Dominique Lemur \\ IETR \\ University of Rennes 1 \\ Rennes, France \\ dominique.lemur@univ-rennes1.fr
}

\begin{abstract}
Internet of Things (IoT) has been scaling up over the last few years in multiple applications and due to the need for geolocation and tracking capabilities, the usage of traditional Time Difference of Arrival (TDOA) arises. In this paper, a novel methodology for localizing using TDoA is presented, after the detailed and complete description of the TDoA has been provided. This proposed method depends on the hyperbolic functions to localize the node on a hyperbola, rather than locating it in a free position in the space potentially suffering from the influence of the timestamp imperfections. Thus, the proposed approach is finding this location on a hyperbola at a point which has the minimum Euclidean distance to all the other hyperbolas. A comparison is performed investigating the attainable accuracies for localizing based on this parametric TDoA and the classical TDoA method, on a well-defined simulation environment. The simulator is based on a Poisson distribution approach for defining the gateways and the node topology, as well as a noise model for emulating the oscillator drift at the gateways. In the given results, the feasibility of the proposed technique is asserted by a drastic improvement over a wide range of drift variances and the number of gateways. This manifests the robustness of the contributed method to the outlier timestamps and its optimum rendering, especially when the number of gateways is expected to be increased in the future.
\end{abstract}

Index Terms-IoT, LoRa, Localization, TDoA, Hyperbolic Function

\section{INTRODUCTION}

The last decade has witnessed the requirement of connectivity everywhere by telecommunication devices beyond the traditional voice, video and data services leading to the birth of the era of the Internet-of-Things (IoT). Unlike traditional telecommunications where data throughput is the main element in the context of the Internet of Things, the focus here is on low-cost deployments in large areas mostly in licence-free frequency bands, such as ISM/SRD (Industrial, Scientific, and Medical/Short Range Device). One main technology for providing this connectivity are Low Power Wide Area Networks (LPWAN), especially the LoRaWAN [1]. This is designed to compromise between energy consumption exhibited in the battery lifetime and communication range of the underlying IoT-enabled devices. Accordingly, the typical LPWAN devices use transmit powers of $10 \mathrm{~mW}$ but are able to cover distances of $10 \mathrm{~km}$ and more while maintaining extremely long battery lifetimes at the scale of up to several years [2].

Positioning is critical for many LPWAN applications because of the very nature of the data collected from the terminals. Examples of these applications are health data, monitoring of pets or livestock, wildlife or applications for smart cities using sensors such as temperature or air quality monitoring in urban environments [4]. A simple solution to this problem would be to equip each sensor with a Global Navigation Satellite Systems (GNSS) chip, for example, using GPS. While this solution is tempting, adding a GPS tracker to a device will increase both cost and power consumption [3]. Thus, it needs to be recharged every few days as the current consumption of a GPS receiver is about $30 \mathrm{~mA}$ to $50 \mathrm{~mA}$, which is considered more energy required by most low power IoT devices. For instance, a LoRaWAN module operating in the $868 \mathrm{mHz}$ band consumes $2.8 \mathrm{~mA}$, in the "on" state, $38.9 \mathrm{~mA}$ transmitting data and $14.2 \mathrm{~mA}$ receiving data [5]. Therefore, the motivation of this work arises from the need of designing a low-power consumption system allowing localization without using GPS. At this point, LoRa plays an important role due to its interesting features which can enable passive localization techniques and also open fields to the new innovative applications that are growing.

In the field of GPS-Free localization in IoT, plenty of researches have been done various investigations to the methods based on Received Signal Strength Indicator (RSSI), Angle of Arrival (AOA), Time of Arrival (TOA), Time Difference of Arrival (TDOA) and their multiple combinations [6]. The gateways utilize these measurements to determine their relative position relations with the source for localization. In RSSI techniques, the existing relation between received power and the node position is exploited [7]. However, these techniques are sensitive to the channel environments and often require a good knowledge of the signal attenuation model [8]. In AOA techniques the angle from which the signal propagates is determined. The maintenance of an antenna array and precise calibration are always required, which result in extra expenses, moreover, their localization accuracy is sensitive to the distances between the node and the gateways [9]. ToA is one of the most accurate techniques 
available as it uses timestamps embedded in transmitted packets along with the received time to determine how far the packet had to travel to reach the destination [10]. Through the use of synchronized clocks, the signal propagation time between the transmitter and receiver can be determined. However, when using a ToA set up, devices in the network need synchronized clocks down to nanosecond scale in order to achieve a proper distance approximation, which requires additional hardware, thus increasing the cost of the system. Therefore, these three kinds of techniques are not so practical.

TDoA is similar to ToA but it is a more popular technique for localization as it does not require the transmitter to be synchronized with the receivers but only the gateways are required to have synchronized clocks [11]. A mobile node transmits data to the network. Each gateway within reach records the time stamp of the received packet. Accurate time stamping is possible since each gateway has a GPS receiver for time synchronization. The timestamps of each gateway are then forwarded to the network server which in turn sends a request to the geolocation solver. After acquiring the differences between the timestamps of a transmission, it is considered a multilateration problem which involves solving a set of hyperbolic functions, and therefore three gateways are needed to locate the node on the intersecting point of the hyperbolas. Based on that, the conventional geolocation for LoRa networks can be based on TDoA measurements, where hundreds to thousands of meters accuracy can be achieved after filtering. However, in a practical situation, the quality of each timestamp can vary largely from one gateway to another. This is due mostly to the time drift which occurs in the timestamping process and the effect of the multipath in Non-line-of-sight (NLOS) situations. These outliers timestamps may have an extremely deleterious effect on the final position accuracy with the classical TDoA solving techniques.

In this paper, to tackle this problem, a novel parametric TDoA method is proposed that uses the hyperbolic parameterization to localize the node on one of the hyperbolas instead of localizing it on the space far from the vicinity of the hyperbolas. The accuracy and ease of use of this algorithm are evaluated through simulations. It is shown that the proposed approach effectively reduces localization errors in a wide range of situations covering different densities of gateways and noise models on ranging data.

The remainder of this document is organized as follows. Section II presents the system model and Section III provides sufficient detail to allow implementation of the proposed algorithm. Section IV provides guidance on the simulation model used to evaluate the performance of the algorithm on the localization accuracy. The results of the simulation are then presented and commented in Section V. Finally, Section VI is dedicated for conclusions.

\section{SYSTEM MODEL}

A set of $N$ gateways are considered with known positions

$$
\mathbf{P}_{g}=\left[\mathbf{p}_{1}, \ldots, \mathbf{p}_{n}, \ldots, \mathbf{p}_{N}\right]
$$

with

$$
\mathbf{p}_{n}=\left[x_{n}, y_{n}\right]^{T},
$$

while $\mathbf{p}=[x, y]^{T}$ is the unknown node position to be localized. The timestamp of the transmitted packet from $\mathbf{p}$ is measured on gateway $n$ as

$$
\tau_{n}=\frac{d_{n}}{c}+u_{n}
$$

where $u_{n}$ is a ranging error term which follows some statistical distribution including several kind of possible errors while, $d_{n}=\left\|\mathbf{p}_{n}-\mathbf{p}\right\|_{2}$ is the distance between the unknown position and the gateway $n$ where, $\|\cdot\|_{2}$ denotes the 2-norm, and $c$ is the celerity of light. Each combination of 2 among $N$ gateways leads to:

$$
H=\left(\begin{array}{c}
N \\
2
\end{array}\right)=\frac{N !}{2 !(N-2) !}=\frac{N(N-1)}{2}
$$

constraints, each being associated with one hyperbola and an hyperbola $j$ involves the 2 gateways $l(j)$ and $r(j)$ while, $\mathbf{l}=[l(1), l(2), \ldots, l(H)]$ and $\mathbf{r}=$ $[r(1), \quad r(2), \ldots, r(H)]$. Accordingly,

$$
\begin{aligned}
\mathbf{P}_{g, \mathbf{r}} & =\left[\mathbf{p}_{r(1)}, \ldots, \mathbf{p}_{r(H)}\right], \\
\mathbf{P}_{g, \mathbf{l}} & =\left[\mathbf{p}_{l(1)}, \ldots, \mathbf{p}_{l(H)}\right]
\end{aligned}
$$

and

$$
\tau_{\mathbf{l}}=\left[\tau_{l(1)}, \ldots, \tau_{l(H)}\right] .
$$

In the classical TDoA techniques, the node location $\hat{\mathbf{p}}=$ $[\hat{x}, \hat{y}]^{T}$ is estimated using the conventional algorithms such as the Least Squares (LS) or by minimizing a cost function on the unknown coordinates [12]:

$$
\begin{array}{r}
\hat{\mathbf{p}}=\underset{x, y}{\arg \min }\left(\sum_{i=1}^{H} \mid \sqrt{\left(x_{l(i)}-x\right)^{2}+\left(y_{l(i)}-y\right)^{2}}\right. \\
\left.-\sqrt{\left(x_{r(i)}-x\right)^{2}+\left(y_{r(i)}-y\right)^{2}}-c\left(\tau_{l(i)}-\tau_{r(i)}\right) \mid\right) .
\end{array}
$$

Hence, the estimated position $\hat{\mathbf{p}}$ could be any point in the plane $\mathbb{R}^{2}$.

\section{PARAMETRIC TDoA}

Unlike the classical TDoA, the main concept of the parametric TDoA is obtaining the point $\hat{\mathbf{p}}_{h, i}$ to be placed on hyperbola $i$ to be determined, by parameterizing the hyperbolic functions. This method is achieved using the following two steps: 
a) Minimizing the Euclidean distance matrix: by letting a set of points, each one lying in a different hyperbola among $H$ hyperbolas to be expressed as:

$$
\begin{aligned}
\mathbf{P}_{h}(\mathbf{t}) & =\left[\mathbf{p}_{h, 1}\left(t_{1}\right), \ldots, \mathbf{p}_{h, H}\left(t_{H}\right)\right] \\
& =\left[\begin{array}{c}
x_{h, 1}\left(t_{1}\right), \ldots, x_{h, H}\left(t_{H}\right) \\
y_{h, 1}\left(t_{1}\right), \ldots, y_{h, H}\left(t_{H}\right)
\end{array}\right],
\end{aligned}
$$

where $\mathbf{t}=\left[\begin{array}{llll}t_{1}, & t_{2}, \ldots, t_{H}\end{array}\right]$ is the vector of the so called hyperbolic angles. Then, finding for every pair of gateways the distance between them as [13]:

$$
\mathbf{d}=\left[\left\|\mathbf{p}_{l(1)}-\mathbf{p}_{r(1)}\right\|_{2}, \ldots,\left\|\mathbf{p}_{l(H)}-\mathbf{p}_{r(H)}\right\|_{2}\right]
$$

and also the difference of distances vector a which is defined for each hyperbola as the timestamps difference at both gateways expressed in meter as:

$$
\mathbf{a}=c\left(\tau_{\mathbf{l}}-\tau_{\mathbf{r}}\right),
$$

while the distances from a focus to either asymptote i.e., the semi-minor axis, are defined as:

$$
\mathbf{b}=\sqrt{\mathbf{d}^{2}-\mathbf{a}^{2}},
$$

as well as, the exact centroid between each pair of gateways are calculated as:

$$
\mathbf{q}=\frac{\mathbf{P}_{g, \mathbf{l}}+\mathbf{P}_{g, \mathbf{r}}}{2} .
$$

Accordingly, all the $H$ points on all the hyperbolas are initially acquired as:

$$
\mathbf{P}_{h}(\mathbf{t})=\mathbf{q}+\frac{\mathbf{a}}{2} \odot \cosh (\mathbf{t}) \odot \mathbf{U}+\frac{\mathbf{b}}{2} \odot \sinh (\mathbf{t}) \odot \mathbf{V}
$$

with

$$
\begin{array}{r}
\mathbf{U}=\frac{\mathbf{P}_{h}\left(t_{\mathbf{r}}\right)-\mathbf{P}_{h}\left(t_{\mathbf{l}}\right)}{\mathbf{d}} \\
=\left[\hat{\mathbf{u}}_{1}, \ldots, \hat{\mathbf{u}}_{H}\right]
\end{array}
$$

and

$$
\mathbf{V}=\left[\hat{\mathbf{z}} \times \hat{\mathbf{u}}_{1}, \ldots, \hat{\mathbf{z}} \times \hat{\mathbf{u}}_{H}\right],
$$

where $\odot$ and $\times$ are defined as the element wise matrix product and the vector cross product, respectively. Based on that, the obtained points $\mathbf{P}_{h}(\mathbf{t})$ have a deterministic Euclidean distance matrix defined as:

$$
\mathbf{D}_{h}(\mathbf{t})=\left[\begin{array}{ccccc}
0 & d_{12}^{2} & d_{13}^{2} & \ldots & d_{1 H}^{2} \\
d_{21}^{2} & 0 & d_{23}^{2} & \ldots & d_{2 H}^{2} \\
d_{31}^{2} & d_{32}^{2} & 0 & \ldots & d_{3 H}^{2} \\
\vdots & \vdots & \vdots & \ddots & \vdots \\
d_{H 1}^{2} & d_{H 2}^{2} & d_{H 3}^{2} & \ldots & 0
\end{array}\right]
$$

where $d_{i j}^{2}=d_{i j}\left(t_{i}, t_{j}\right)^{2}=\left\|\mathbf{p}_{h, i}\left(t_{i}\right)-\mathbf{p}_{h, j}\left(t_{j}\right)\right\|_{2}^{2}$ is the Euclidean distance between the hyperbola $i$ and $j$. Here, the main target is to find the proper hyperbolic angles $\mathbf{t}_{\text {min }}$ which minimize the summation of the Euclidean distance matrix $\mathbf{D}_{h}(\mathbf{t})$, and this can be achieved using a solver as:

$$
\mathbf{t}_{\text {min }}=\underset{\mathbf{t}}{\arg \min }\left(\sum_{i=1}^{H} \sum_{j=1}^{H} d_{i j}\left(t_{i}, t_{j}\right)^{2}\right)
$$

b) Electing the convenient point: from the set of estimated points $\mathbf{P}_{h}\left(\mathbf{t}_{\text {min }}\right)$ is the final step i.e., one of the points among the small cross blue markers as shown in Figure 1. This is achieved by picking the index of the point which has the minimum Euclidean distance to all the other hyperbolas as:

$$
i_{\text {min }}=\underset{i}{\arg \min }\left(\sum_{j=1}^{H} d_{i j}\left(t_{m i n, i}, t_{m i n, j}\right)^{2}\right) .
$$

Hence, the final estimated location of the node is considered as:

$$
\hat{\mathbf{p}}_{h, i_{\text {min }}}=\mathbf{p}_{h, i_{\text {min }}}\left(t_{m i n, i_{\text {min }}}\right),
$$

which is the large cross blue marker in Figure 1, actually most of the cases closer to the true position than the classical TDoA estimation with the large cross green marker. The reason behind the proposed method is that should be less sensitive to the outliers because of the constraint imposed on the points of belonging by construction to hyperbolas associated with the constraints.

\section{Simulation Model}

In this section, a brief overview of the simulator model is given in order to allow for a dynamic study of the system performance. Thus, the two main aspects shaping the simulation scenarios are the distributions of the gateway locations with respect to the node location and the choice of convenient uncertainty in the timestamps, to be closer to the real measurements as detailed in the following subsections.

\section{A. Gateway locations}

The gateway locations are produced by the Poisson disk distributions algorithm which has been introduced in [14]. This is considered as a fast 2-dimensional blue noise sampler, easily implemented in arbitrary dimensions and it is guaranteed to take $O(M)$ time to generate $M$ Poisson disk samples. To start the process, this algorithm takes as input the length len and width wid of the samples domain in $\mathbb{R}^{2}$, and the minimum distance $\rho$ between the samples. First, it initializes a 2-dimensional background grid for storing samples and accelerating the spatial searches. Then, it selects the initial sample randomly chosen uniformly from the domain and inserts it into a cell in the background grid. The cell size is picked to be bounded by $\frac{\rho}{\sqrt{2}}$, so that each grid cell will contain at most one sample. In the next iteration, the neighboring point is chosen uniformly from the spherical annulus between radius $\rho$ and $2 \rho$ around the previous sample. This linear algorithm is done recursively until all the $M$ samples are generated. At this point, the 
node location is chosen randomly from the samples, while maintaining the other samples to be the gateway positions as shown in Figure 1.

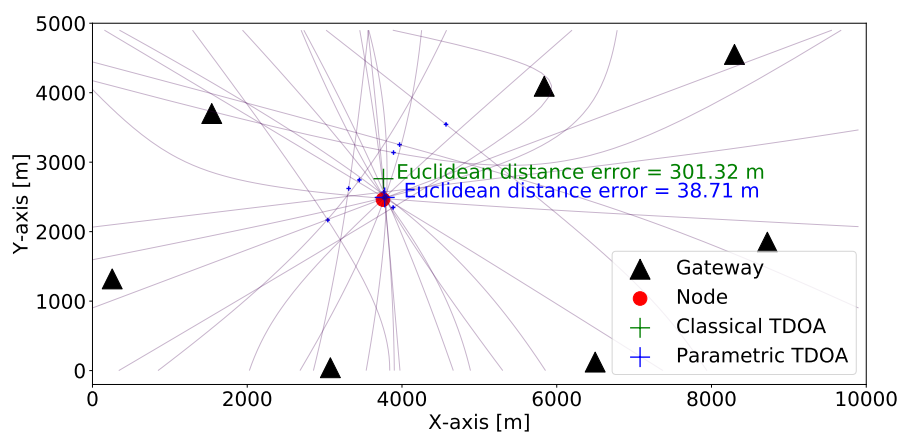

Fig. 1: A sample pattern from the algorithm $(\rho=2.5 \mathrm{~km}$, wid $=10 \mathrm{~km}$, len $=5 \mathrm{~km}$ ).

\section{B. Timestamp perturbation model}

From our knowledge of what is observed in LoRaWAN practical situations, it often happens that few gateways are suffering from much larger uncertainty than the others. To model this fact, a Gaussian mixture distribution is proposed. The first Gaussian is taking into account the measurement noise whereas the second is taking into consideration the larger oscillator drift perturbation. Hence, the timestamp is individually calculated after extending Equation 3 by modeling the uncertainty term $u_{n}$ as:

$$
\tau_{n}=\frac{d_{n}}{c}+u w_{1}+(1-u) w_{2}
$$

with

$$
\operatorname{Pr}(u=1)=p=1-\operatorname{Pr}(u=0),
$$

where $w_{1} \sim \mathcal{N}\left(0, \sigma_{1}^{2}\right)$ and $w_{2} \sim \mathcal{N}\left(0, \sigma_{2}^{2}\right)$ are the normal Gaussian distributions of the thermal noise and the gateway oscillator drift, respectively, by assuming zero means in both cases. Thus, the probability $p$ is chosen equal to 0.8 taking into account the occurrence probability of the oscillator drift by $20 \%$.

\section{Simulation Results}

In the previous section, the simulation framework is provided including the location distributions and the chosen noise model. In this section, the simulation results are presented, using the Euclidean distance error between the true position of the node and the estimated location, as a measure of performance for the localization methods. This is measured by Monte Carlo simulations after utilizing the proposed parametric TDoA technique and the classical TDoA using LS i.e., introduced in [12], for comparison. Moreover, the two main parameters which shape the simulation scenarios are the magnitude of the oscillator drift variance and the number of gateways as detailed in the following subsections.

\section{A. Impact of the oscillator drift variance}

The robustness of the system against the outlier timestamps is checked by changing the drift standard deviation $\sigma_{2}$ in Equation 21 to be in the range from $0 \mu \mathrm{s}$ to $2 \mu \mathrm{s}$ ( $\equiv 0 \mathrm{~m}$ to $600 \mathrm{~m}$ ), while maintaining the thermal noise standard deviation $\sigma_{1}$ to be equal to $0.1 \mu \mathrm{s}$ ( $\equiv 30 \mathrm{~m}$ ). For the gateway location distribution, the length len and width wid of the map are fixed to $5 \mathrm{~km}$ and $10 \mathrm{~km}$, respectively, while the minimum distance $\rho$ between the points is equal to $2.5 \mathrm{~km}$. This distribution configuration usually gives a median number of gateways between 5 and 8 .

As shown in Figure 2a, the simulation result shows an obvious reduction in the localization error medians when using the parametric TDoA, especially for the large values of the drift. This indicates that the proposed method is more robust to the high drifts. Accordingly as shown in Figure $2 b$, the CDF curves obtained for all the drift values preserve the same performance rank over the whole simulations with $50 \%$ of the error values less than $40 \mathrm{~m}$ and $65 \mathrm{~m}$, using the proposed parametric and classical TDoA, respectively.

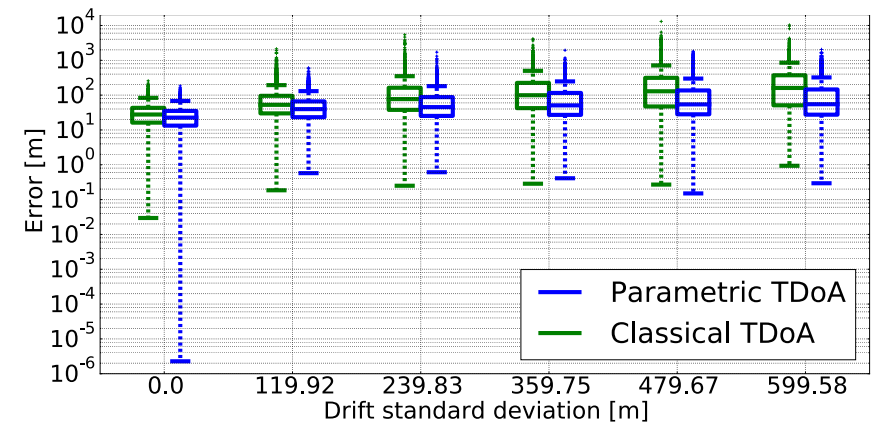

(a) Errors at each value of the drift standard deviation.

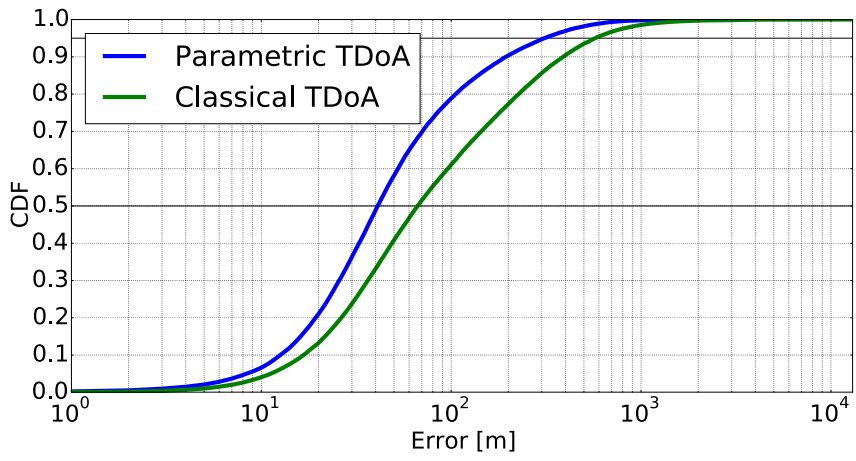

(b) The whole simulation results of $\sigma_{2}$ ranging from $0 \mathrm{~m}$ to $600 \mathrm{~m}$.

Fig. 2: Euclidean distance error and CDF while changing drift standard deviation $\sigma_{2}$.

\section{B. Impact of the number of gateways}

The localization performance assessment for various number of gateways is studied by utilizing the minimum distance $\rho$ between the gateways to be in the range from 
$1.6 \mathrm{~km}$ to $4 \mathrm{~km}$, while fixing the length len and width wid of the map to be $5 \mathrm{~km}$ and $10 \mathrm{~km}$, respectively. Moreover in all the results, the drift standard deviation $\sigma_{2}$ and the thermal noise standard deviation $\sigma_{1}$ are assumed to be equal to $1.2 \mu \mathrm{s}$ $(\equiv 360 \mathrm{~m})$ and $0.1 \mu \mathrm{s}(\equiv 30 \mathrm{~m})$, respectively.

As shown in Figure 3a, it is clear that all the medians of the parametric method are drastically the lowest values for all the network densities. While by observing the extreme error values, the main parameter in achieving the performance promised by the proposed algorithm without these outlier values, is the large number of gateways $(>4)$ i.e., a realistic value in the near future. Thus, it is clear in the lower network density that the number of hyperbolas decreases accordingly, resulting in an incorrect selection of the convenient point i.e., discussed in Section II, from the set of estimated points $\mathbf{P}\left(\mathbf{t}_{\text {min }}\right)$ and a less accurate localization. Only at these low number of gateways, there is a trade-off to find between the two methods, the parametric one being better regarding the median and worst regarding the outliers. Nevertheless as shown in Figure $3 b$, the CDF curves obtained for all the inner radius values still confirm the prevalence of the proposed method over most of the simulations with $95 \%$ of the error values less than $840 \mathrm{~m}$ and $2200 \mathrm{~m}$, using the proposed parametric and classical TDoA, respectively.

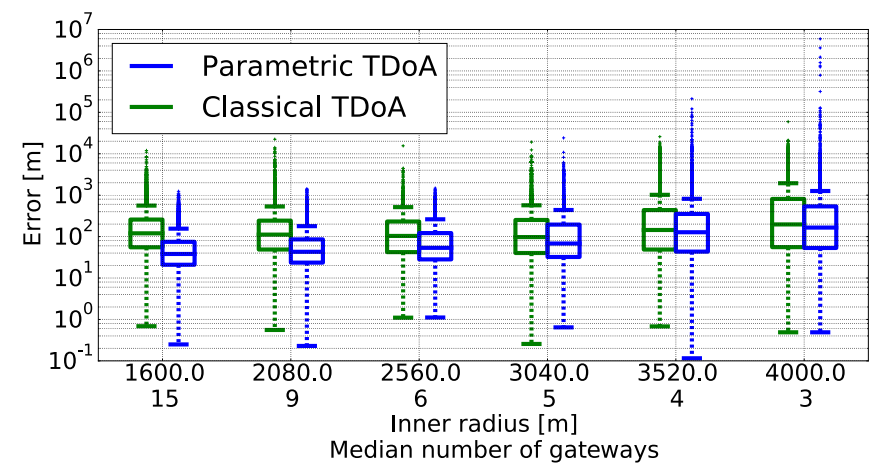

(a) Errors at each number of gateways.

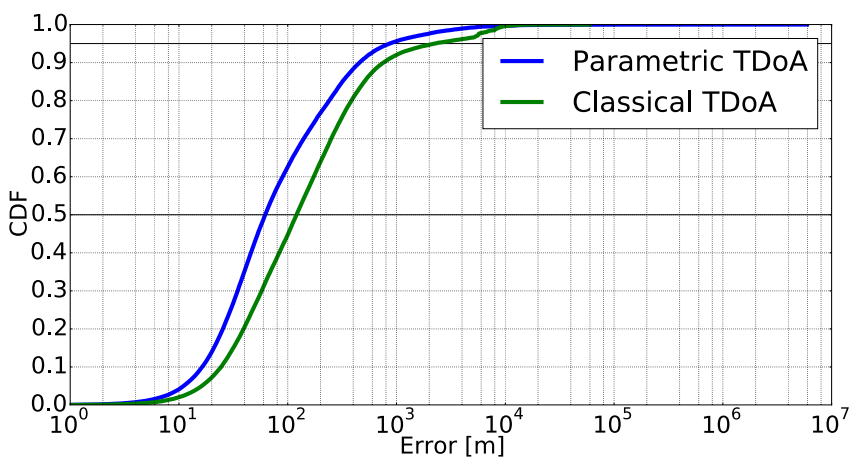

(b) The whole simulation results of $\rho$ ranging from $1.6 \mathrm{~km}$ to $4 \mathrm{~km}$.

Fig. 3: Euclidean distance error and $\mathrm{CDF}$ while changing inner radius $\rho$.

\section{CONCLUSIONS}

In this article, a novel TDoA parametric method for IoT localization is presented. The technique improves the accuracy of localization in the presence of outliers, even when high levels of drift in gateways can hardly be avoided. The principle is based on the choice of the node position to be located on one of the hyperbolas by bringing the optimization into the parametric space of the hyperbolic constraint sets. For performance assessment of this technique, a simulator has been developed that uses a Poisson distribution approach for determining the location of gateways and nodes based on configurations chosen to be as close as possible of realistic situations encountered in the LoraWan context. Moreover, a noise model is proposed to emulate the proper disturbance in the timestamp values by considering the drift variance. Simulation results prove the high performance of our parametric method over a wide range of drift standard deviations and network densities. In some configurations the achieved performance using the proposed technique, almost matches the performance in non-drifted timestamp scenarios. This competes particularly well regarding localization accuracy with more traditional approaches. This is going to become increasingly useful when situations of localization with a greater number of gateways will be more frequent in the future.

In future work, parametric TDoA can be used to detect outliers as well as an initial guess point for more sophisticated localization approaches that integrate other a priori information. The presented TDOA technique can be complemented with classical machine learning techniques for merging other radio observations such as RSSI, angular estimates or digital elevation model of the propagation environment.

\section{REFERENCES}

[1] M. Centenaro, L. Vangelista, A. Zanella and M. Zorzi, "Long-range communications in unlicensed bands: the rising stars in the IoT and smart city scenarios," in IEEE Wireless Communications, vol. 23, no. 5, pp. 60-67, October 2016.

[2] F. Adelantado, X. Vilajosana, P. Tuset-Peiro, B. Martinez, J. Melia-Segui and T. Watteyne, "Understanding the Limits of LoRaWAN," in IEEE Communications Magazine, vol. 55, no. 9, pp. 34-40, Sept. 2017.

[3] A. H. Sayed, A. Tarighat and N. Khajehnouri, "Network-based wireless location: challenges faced in developing techniques for accurate wireless location information," in IEEE Signal Processing Magazine, vol. 22, no. 4, pp. 24-40, July 2005.

[4] L. Mainetti, L. Patrono, A. Secco and I. Sergi, "An IoT-aware AAL system for elderly people," 2016 International Multidisciplinary Conference on Computer and Energy Science (SpliTech), Split, 2016, pp. 1-6.

[5] Libelium Comunicaciones Distribuidas S.L., "Waspmote LoRaWAN Networking Guide," v7.0, pp. 5-11, October 2016.

[6] S. Sadowski and P. Spachos, "RSSI-Based Indoor Localization With the Internet of Things," in IEEE Access, vol. 6, pp. 30149-30161, 2018.

[7] Z. Li, T. Braun, X. Zhao, Z. Zhao, F. Hu and H. Liang, "A Narrow-Band Indoor Positioning System by Fusing Time and Received Signal Strength via Ensemble Learning," in IEEE Access, vol. 6, pp. 9936-9950, 2018.

[8] Choi, Wongeun and Chang, Yoon-Seop and Jung, Yeonuk and Song, Junkeun. (2018). Low-Power LoRa Signal-Based Outdoor Positioning Using Fingerprint Algorithm. ISPRS International Journal of GeoInformation. 
[9] T. Sathyan, A. Sinha and T. Kirubarajan, "Passive geolocation and tracking of an unknown number of emitters," in IEEE Transactions on Aerospace and Electronic Systems, vol. 42, no. 2, pp. 740-750, April 2006.

[10] Zafari, F., Gkelias, A., and Leung, K. K. (2019). A Survey of Indoor Localization Systems and Technologies. IEEE Communications Surveys and Tutorials, 1-1

[11] B. C. Fargas and M. N. Petersen, "GPS-free geolocation using LoRa in low-power WANs," 2017 Global Internet of Things Summit (GIoTS), Geneva, 2017, pp. 1-6.

[12] Alan Bensky. 2007. Wireless Positioning Technologies and Applications. Artech House, Inc., Norwood, MA, USA.

[13] Mitchell, Douglas W., "A property of hyperbolas and their asymptotes", Mathematical Gazette 96, July 2012, 299-301.

[14] Robert Bridson. 2007. Fast Poisson disk sampling in arbitrary dimensions. In ACM SIGGRAPH 2007 sketches (SIGGRAPH '07). ACM, New York, NY, USA, , Article 22 . 